\title{
Computational Modeling of Common Failures in Electrical Induction Machines
}

\author{
Adrian Gonzalez-Parada ${ }^{1}{ }^{*}$, Rafael Guzmán-Cabrera ${ }^{1}$, Miguel Torres-Cisneros ${ }^{1}$, Ricard \\ Bosch-Tous ${ }^{2}$, Hector J. Estrada-García ${ }^{1}$ \\ ${ }^{1}$ Engineering Division, University of Guanajuato, México. \\ ${ }^{2}$ School of Industrial Engineering of Barcelona (ETSEIB), Polytechnic University of Catalonia, Barcelona, \\ Spain. \\ * Corresponding author. Tel.: +52 (464) 6479940 2354; email: gonzaleza@ugto.mx \\ Manuscript submitted October 5, 2015; accepted December 13, 2015. \\ doi: 10.17706/ijcee.2015.7.6.417-423
}

\begin{abstract}
One of the most common types of failures in induction motors with squirrel cage rotor is examined in this paper. Generally, such failure is characterized by: broken rotor bars and short circuit in the inter-turn winding. The broken rotor bars failure was simulated with two consecutive broken bars in order to see how the magnetic flux density within be affected. The inter-turn short circuit was simulated with $40 \%$ reduction of winding coil in an inter-turn short circuit for one of the phases. In both cases the simulation was performed with the finite element software FEMM ${ }^{\odot}$, which helped in obtaining the waveform of the flux density in the motor airgap and the magnetic core. The result obtained allows clear identification of the type of failures that occurs depending of type that will present in the simulation.
\end{abstract}

Key words: Computation modeling, emerging faults, electrical machines, failure detection.

\section{Introduction}

Motors are commonly used in a variety of industrial applications, and some induction motors are key devices in assuring the continuity of processes and the production chains of many industries. However, the failure in electrical motors may be attributed to three main reasons, which cause the most common failures.

Studies carried out by the Electric Power Research Institute (EPRI) and the General Electric has shown the causes of failures in induction motors [1]. The most common failures in AC motors were found to be related to the bearing (41\%): this kind of failures are easily identifiable due to an overwhelming fingerprint: the noise when these failures occur. The second most common failure (37\%) in motors occurs in the stator, this failure is quite more difficult to identify because it takes place inside the winding. Finally, the third most common failure in AC motors (10\%) is the breakage of the rotor bars. This kind of failure is quite difficult to identify in comparison to stator failure, not only because special detection equipment is required but because the motor has to be switched out of line so that the corresponding tests can be performed.

All these failures have been widely studied and several methods have been developed in order to improve detection techniques [2], [3]. In this work we propose a hybrid technique of using finite element simulation and computational algorithms to model, process, and analyze electrical signals related to failures of intern-turn short circuit in the winding and the rotor broken bars. The obtained results form a set of characteristics that later allow performing reliable detection, diagnosis, and discrimination between these two kind of failures. 


\section{Theoretical Background}

\subsection{Stator Winding Failure}

If a short circuit occurs in the turns of the winding a new path for current circulation is formed. This new path causes a decrease in the coil impedance of the winding due to the reduction of the coil turns (i.e. turns that were in series are now connected in parallel). The main consequence of this kind of failure is the increment of the total current flowing through the shorted circuit winding. This additional current causes a temperature increase and then progressive failure of machine insulation.

Many works that can be found in the literature approach the problem in terms of analysis of airgap and axial flux signals in order to detect shorted turns in the winding: the mathematical formulations can be found in references [4], [5]. Previous works have demonstrated that the following equation gives the components in the air gap flux waveform as a function of shorted inter-turns.

$$
f_{s t}=f_{s f}[n / p(1-s) \pm k]
$$

where $f_{s t}$ is the spectral component indicating turn-to-turn faults, $f_{s f}$ is the supply frequency, $n=1,2,3, \ldots, k=$ $1,3,5, \ldots, p$ is the number of pole pairs and $s$ is the slip. Due to the presence of voltage unbalances and other asymmetries, only frequencies corresponding to specific combinations of $k$ and $n$ in (1) can be used for short-circuit-fault diagnosis, where the standard method is to perform frequency analysis using Fourier transforms.

Inter-turn short circuits have a cumulative effect in decreasing MMF in the vicinity of short-circuited turns. First, when a short circuit occurs, the damage phase winding as less turns and therefore less MMF. Secondly, the short-circuited turns create their own MMF but in opposition of phase with that of the injured phase [6].

\subsection{Rotor Bar Failure}

Previous works have demonstrated that broken bars can be a serious problem with certain induction motors because of their arduous duty cycles [7]. Although broken rotor bars do not initially cause a motor failure there can be serious secondary effects. This can cause serious mechanical damage to the insulation and consequently winding failures may follow, resulting in costly repair and production loses.

Many works previously developed to analyze the theoretical performance of three-phase induction motors with broken rotor bars based on their analysis on the symmetric magnetic field supply by the stator [8], [9]. The rotor current in a cage winding produce an effective three-phase magnetic field rotating at slip frequency $\left(f_{2}\right)$ with respect to the rotating rotor. When the cage winding is symmetrical, there is only a forward rotating field at slip frequency with respect to the rotor. The occurrence of a rotor asymmetry will result in a backward rotating field at slip frequency with respect to the forward rotating rotor. The overall effect resulting from the procedure is the induction of an EMF and current in the stator winding at frequency:

$$
F_{B B}=f_{1}[n / p(1-s) \pm k]
$$

where $F_{B B}$ is the slip frequency sideband with respect to the rotor, $f_{1}$ is the supply frequency (Hz), $s$ is per unit slip and $k=1,3,5$. This is referred to as a twice slip frequency sideband due to the broken rotor bars. Therefore, a cyclic variation of the current causes a torque pulsation at twice the slip frequency ( $2 s f_{1}$ ) and the corresponding speed oscillation that is also a function of the drive inertia. This speed oscillation can reduce the magnitude of the $f_{1}(1-2 s)$ sideband but an upper sideband current component at $f_{1}(1+2 s)$ is 
induced in the stator winding due to rotor oscillation [9]. This upper sideband is also enhanced by the third harmonic flux.

\section{Simulation and Tests}

\subsection{Finite Element Analysis (FEA)}

Computer simulation using machine models facilitates the study of short-circuit faults in electrical machines. It provides an inexpensive and convenient method for initial development and testing of a fault-diagnosis algorithm. Machine models offer the additional flexibility of simulating different machines by changing the parameters of the model.

The physical systems are frequently represented by the partial differential equations (PDE) systems associated to boundary conditions in space and time. FEM allows a discrete representation of these nonlinear equations converting them into an algebraic equations system. If the magnetic field is time-varying, eddy currents can be induced in materials with non-zero conductivity. Therefore, the time-varying field equation can be used for modeling the induction machine, which has eddy current. The Maxwell flux equation for time-harmonic magnetic problems is given below:

$$
\nabla \times\left(\frac{1}{\mu} \nabla \times A\right)=J-\sigma d t A
$$

where $A(\mathrm{~Wb} / \mathrm{m})$ is the magnetic vector potential, $J\left(\mathrm{~A} / \mathrm{m}^{2}\right)$ is the total current density vector, $\mu(\mathrm{H} / \mathrm{m})$ is the magnetic permeability and $\sigma(\mathrm{S} / \mathrm{m})$ is the electric conductivity.

The numerical simulations of the induction motor were performed by finite elements in order to obtain graphical representations of the magnetic and electric fields distributions. This kind of simulations also allows in knowing the electric and magnetic field values precisely in each section of the motor so that qualitative and quantitative comparisons between failure and non-failure conditions can be carried out. The electrical machine analyzed is an induction motor with squirrel cage rotor whose characteristics are shown in Table 1.

Table 1. Induction Motor Characteristics

\begin{tabular}{ll}
\hline \hline Motor Characteristics: & Data \\
\hline Power: & $7.5 \mathrm{HP}$ \\
Connection: & $220 \mathrm{~V}$ Double star \\
Slots/coil: & $1-7$ \\
Turns: & 59 \\
Coils/Group: & 2 \\
Slots: & 24 \\
Voltage: & $208-230 / 460$ \\
Amp: & $3.2-3 / 1.5$ \\
Magnetic core length: & $2 " 1 / 2$ \\
Magnetic core diameter: & 3 "1/8 \\
Magnetic core inner diameter: & 1 "9/16 \\
Magnetic core outer diameter: & 2 "12/16 \\
\hline \hline
\end{tabular}

In order to simulate the winding's section, 3 electric circuits were created; each circuit corresponds to a phase of the motor supply. In each of these circuits a current (i.e. 1.5 A.) and phases (i.e. $0^{\circ}, 120^{\circ},-120^{\circ}$ ) of corresponding current of an electric system in a polar configuration were implemented. In the simulation, the motor winding was connected in double star in order to obtain a connection to $220 \mathrm{~V}$. A general drawing 
of the motor was done taking into consideration a general winding distribution and the real dimensions of the rotor bars.

Comparative results of motor performance three representative simulations were performed. The first one refers to a motor without failure; the second one represents a motor with broken rotor bars; and the third simulation represents a motor with $40 \%$ short circuit inter-turn in one phase one of the winding.

\subsection{Simulation of a Normal Motor}

The simulation of the motor without failure was developed according to the general specifications for the motor and the materials mentioned above. The results obtained from the simulation for the flux density and the flux lines inside stator core are shown in Fig. 1. The magnetic poles as well as the uniform magnetic flux density around the motor are clearly seen and distortion of flux in the air gap remains unseen and the stator is observed (see Fig. 1(a)).

The analysis of the magnetic flux in the air gap between rotor and stator could be made considering performance of the magnetic field in the middle. The magnetic field distribution in the air gap can be observed in Fig. 1(b), this distribution is uniform throughout the section analyzed, the distortion observed is due to de harmonic performance of the magnetic flux in the airgap between the rotor and the stator.

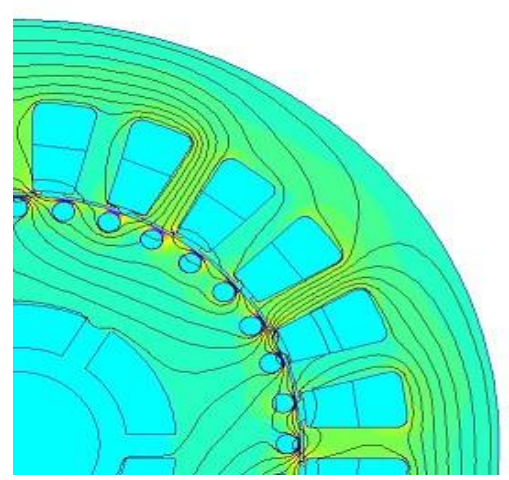

(a)

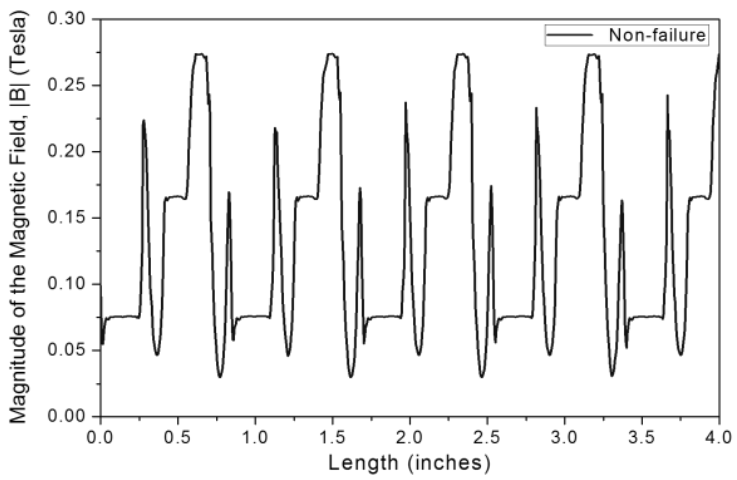

(b)

Fig. 1. Magnetic field distribution of a motor without failure, (a) Simulation of Magnetic flux distribution, (b) Magnetic field distribution in the airgap.

\subsection{Simulation of Motor with Inter-Turn Short Circuited}

In order to simulate the failure in the inter-turn, one group of the winding was decreased by $40 \%$ for one phase. The results obtained for the inter-turn failure simulation for the phase A are shown in Fig. 2. In similar fashion as the previous case, the regions where the failure is induced have been marked with circles; the slots enclosed within the circles are the failure zone for the phase A with a $40 \%$ turn reduction in the winding. The flux density distribution in the core can be observed with the graphical distribution along the airgap in the failure zone.

The magnetic flux distribution along the airgap is affected by the inter-turn reduction in the winding, as it is observed in Fig. 4(b). The current density variation induced in the rotor causes a notorious imbalance due to the fault. As a consequence a low magnetic flux density is generated and less current is induced in the fault zone of the rotor.

\subsection{Simulation of Broken Bars in the Rotor}

The broken bar condition in an induction motor with a squirrel cage rotor can be simulated considering the current absence in the bars analyzed. In this case, two consecutive bars were selected for simulation 
purposes. The materials and motor specifications were the same as those mentioned above. The results obtained are shown in Fig. 3, the location where the failure is being induced has been marked with the circle (see Fig. 3(a)). The lines of magnetic field distribution in the airgap present a distortion due to the broken bars and leads to an increase in magnetic flux within the failure zone; this distortion was highlighted in Fig. 3(b).

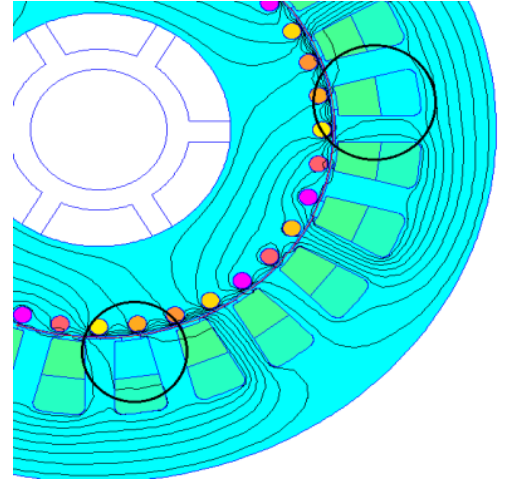

(a)

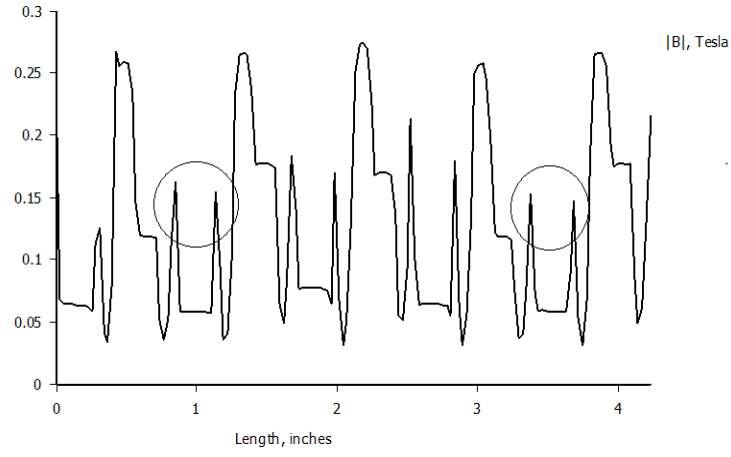

(b)

Fig. 2. Simulation and magnetic flux distribution of the inter-turn failure on the winding. (a) FEM solution for $40 \%$ reduction, (b) Magnetic field performance in the airgap.

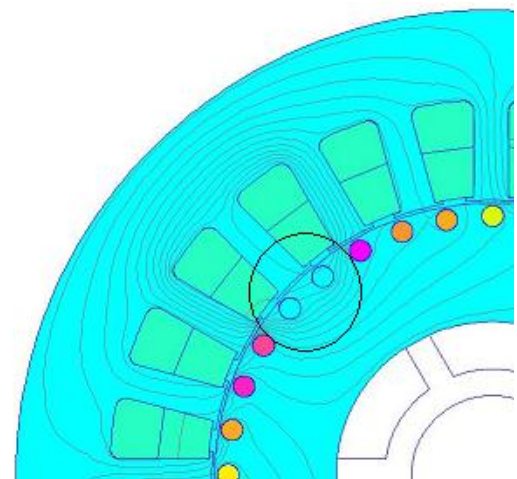

(a)

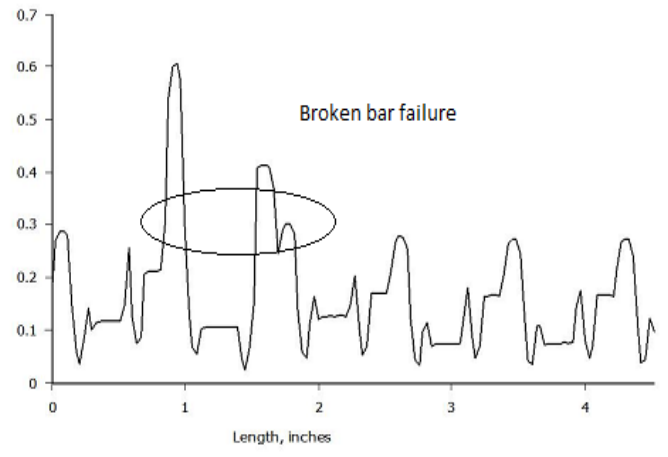

(b)

Fig. 3. Magnetic field distribution in an induction motor with two broken bars. (a). Two broken bars and its Magnetic flux distribution, (b) Magnetic field performance in the airgap.

In order to verify of the simulations results, test of electric motors with failures caused intentionally in the stator and rotor were made, comparing the results with an electric motor in good condition.

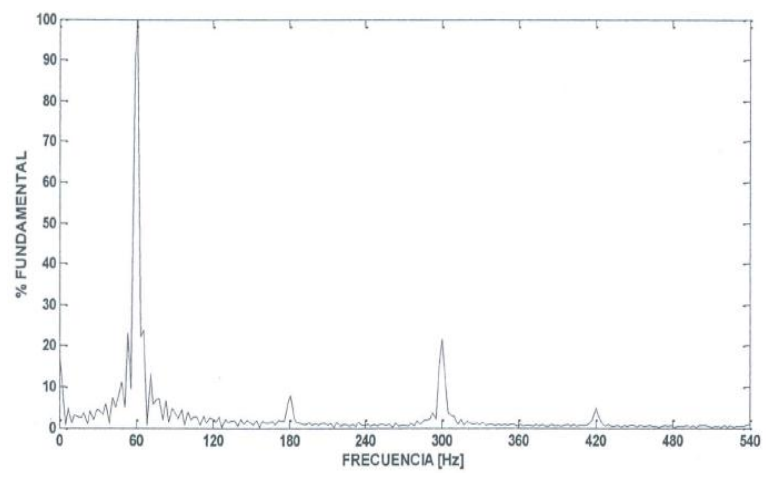

(a)

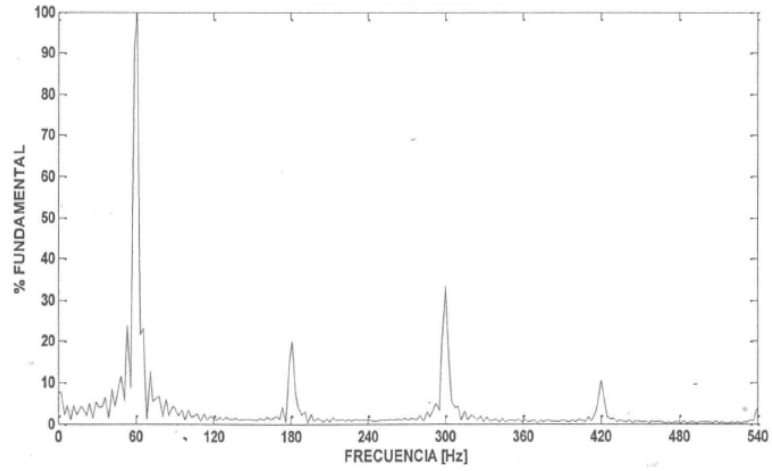

(b)

Fig. 4. Comparative analysis frequency of the circulating current of a motor in good condition and failure. (a) Motor in good condition, (b) Motor with inter-turn failure. 
The circulating current was measured in the motor in good condition and failure, performing a frequency analysis with FFT finding variations on the 3rd, 5th and 7th harmonic. The Fig. 4 shows the results obtained for a) Motor in good condition and b) motor whit inter-turn failure on the winding. Similar performance was obtained for a motor with broken bars.

\section{Conclusion}

There have been simulating the most common types of failures that occur in the induction motors and clearly identified the occurrence of distortions in the measured flow in the air gap, this can be used to simulate the behavior of the electrical motors in performance test and failure analysis.

The distortion introduced in the air gap magnetic flux can be detected analyzing on frequency the motor current, where they will have variations in the harmonic distortion of the current flowing through the stator. This can be implemented by a data acquisition system in real time being able to anticipate catastrophic machine failure.

\section{References}

[1] IEEE Motor Reliability Working Group. (1985). Report of large motor reliability survey of industrial commercial installations, part I. IEEE Transactions on Industrial Applications, 21, 853-872.

[2] Thomson, W. T., \& Fenger, M. (2001). Current signature analysis to detect induction motor faults. IEEE INDUSTRY Application Magazine, 26-34.

[3] Talam, R. M., Lee, S. B., Stone, G., Kliman, G. B., Yoo, J., Habetler, T. G., Harley, R. G. (2003). A survey of methods for detection of stator related faults in induction machines. Proceedings of IEEE International Symposium on Diagnostics for Electrical Machines, Power Electronics and Drivers (pp. 35-46). Atlanta, USA.

[4] Sarma, M. (1996). Electric Machines: Steady-State Theory and Dynamic Performance (2nd ed.). PWS Publishing Company.

[5] Stavrou, A., Sedding, H., \& Penman, J. (2001). Current monitoring for detecting inter-turn short circuit in induction motors. IEEE Transactions on Energy Conversion, 16, 32-37.

[6] Sahraoui, M., Ghggal, A., \& Zouzou, S. E. Modelling and detection of inter-turn short circuits in stator windings of induction motors.

[7] Thomson, W. T., \& Barbour, A. (1998). On-line current monitoring and application of a finite element method to predict the level of static airgap eccentricity in three-phase induction motors. IEEE Transactions on Energy Conversion, 13(4), 347-357.

[8] Jung, J. H., Lee, J. J., \& Kwon, B. H. (2006). Online diagnosis of induction motors using MCSA. IEEE Transaction on Industrial Electronics, 53(6), 1842-1852.

[9] Faiz, J., Ebrahimi, B. M., Akin, B., \& Toliyat, H. A. (2008). Finite element transient analysis of induction motors under mixed eccentricity fault. IEEE Transaction on Magnetics, 44(1), 66-74.

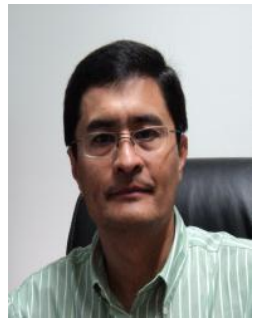

Adrián González-Parada obtained electrical engineering degree from the Queretaro Technological Institute, a master degree in electrical engineering specializing in high voltage from DICIS, University of Guanajuato, Mexico and a PhD degree in electrical engineering from the Polytechnic University of Catalonia in Barcelona, Spain. He is a research-professor in the DICIS of the University of Guanajuato and responsible of the applied superconductivity and special electrical machines. Dr. González-Parada's areas of interest are applied superconductivity in electrical equipment, efficient use of energy in electrical power systems and electrical equipment reliability in the electrical grid. 


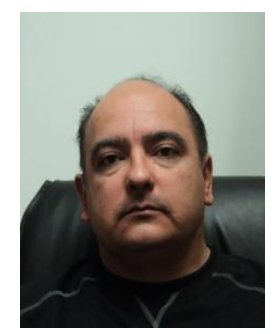

Rafael Guzmán-Cabrera is a full time professor with the Faculty of Engineering Mechanics Electrics and Electronics, Guanajuato University, he obtained a PhD degree in pattern recognition and artificial intelligence from Polytechnic University of Valencia, Spain. He is a contributor in research projects in the area of electrical engineering, pattern recognition and artificial intelligence. He has published over hundred scientific papers, holds 2 patents and he has been adviser more of 40 graduate and undergraduate students.

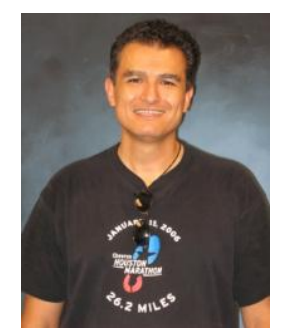

Miguel Torres-Cisneros obtained his engineering degree in electronics at the Universidad de Guanajuato in 1988, his M.Sc. degree from the Centro de Investigaciones en Óptica in 1991 and his Ph.D. degree from the Instituto Nacional de Astrofísica, Óptica y Electrónica in 1997. He has been a visiting research scientist in Dayton University in 2002 and the University of Central Florida, UCF in 2009. He has been a professor at the Tech. of Monterrey and the Universidad de las Américas, and titular researcher at the Universidad de Guanajuato for 17 years, where he is involved with the NanoBioPhotonics Group, the Patents Group, and the Design and Manufacture Cell, Electronics \& Mechatronics Programs.

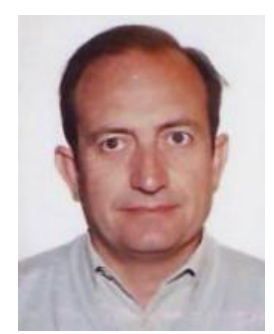

Ricard Bosch-Tous received a PhD of industrial engineering from de Polytechnic University of Catalonia, he has more than 15 years' experience in the electrical equipment field and power electronic in the iron and steel industry. Since 1984, he is a fulltime professor in the Electric Department of the ETSEIB-UPC. Dr. Bosch-Tous is the chief of the High Voltage, Motors, Generators and Special Machines Laboratory in the ETSEIB-UPC.

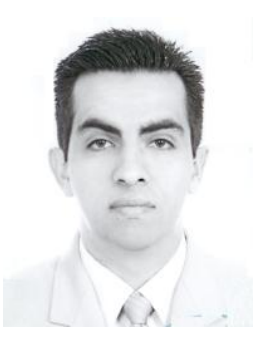

Héctor J. Estrada-García received his Ph.D. degree from CICESE, Mexico in 2008. His principal areas of interest are nonlinear control, smart grids, electrical systems and time delay systems. Currently he is the Electrical Engineering Department head at DICIS, University of Guanajuato, Mexico. 